\title{
B3GNT3: A prognostic biomarker associated with immune cell infiltration in pancreatic adenocarcinoma
}

\author{
KAIWEN KONG $^{1 *}$, YUANYU ZHAO $^{2 *}$, LEILEI XIA $^{3 *}$, HUI JIANG $^{1}$, MINGJUAN XU $^{3}$ and JIANMING ZHENG $^{1}$ \\ ${ }^{1}$ Department of Pathology, Changhai Hospital; ${ }^{2}$ Department of Organ Transplantation, Changzheng Hospital; \\ ${ }^{3}$ Department of Obstetrics and Gynecology, Changhai Hospital, Navy Medical University, Shanghai 200433, P.R. China
}

Received March 4, 2020; Accepted September 28, 2020

DOI: $10.3892 / \mathrm{ol} .2020 .12420$

\begin{abstract}
Pancreatic cancer, one of the most malignant gastrointestinal tumors, is prone to liver metastasis. However, due to the lack of appropriate and comprehensive diagnostic methods, it is difficult to accurately predict the survival outcomes. Therefore, there is a need to identify effective biomarkers, such as UDP-GlcNAc: $\beta$ Gal $\beta-1,3-\mathrm{N}$-acetylglucosaminyltr ansferase 3 (B3GNT3), that predict the survival outcome of patients with pancreatic cancer. In the present study, based on data from 171 cases of pancreatic cancer obtained from The Cancer Genome Atlas portal, the differential expression of mRNAs was screened by comparing cancerous tissues with adjacent tissues. Univariate Cox regression analysis demonstrated that B3GNT3 had prognostic capability and could be an independent prognostic factor for pancreatic adenocarcinoma (PAAD). Using the Tumor Immune Estimation Resource tool and Tumor-Immune System Interaction Database, a potential relationship between B3GNT3 expression and immune cell infiltration was identified in pancreatic carcinoma. Furthermore, 177 samples of pancreatic carcinoma were collected and the association of CD68 expression with B3GNT3 was assessed by immunohistochemical staining. B3GNT3 expression was associated with clinical outcomes in pancreatic carcinoma and related to infiltrating levels of immune cells, which indicated that B3GNT3 could be used as an immunotherapy target for PAAD.
\end{abstract}

Correspondence to: Professor Mingjuan $\mathrm{Xu}$, Department of Obstetrics and Gynecology, Changhai Hospital, Navy Medical University, 168 Changhai Road, Yangpu, Shanghai 200433, P.R. China

E-mail: 13636373419@163.com

Professor Jianming Zheng, Department of Pathology, Changhai Hospital, Navy Medical University, 168 Changhai Road, Yangpu, Shanghai 200433, P.R. China

E-mail: jmzheng1962@163.com

${ }^{*}$ Contributed equally

Key words: UDP-GlcNAc: $\beta$ Gal $\beta-1,3-\mathrm{N}$-acetylglucosaminyltransfe rase 3, pancreatic cancer, biomarker, survival, immune

\section{Introduction}

Pancreatic adenocarcinoma (PAAD) is an aggressive type of malignancy, characterized by rapid progression and dismal prognosis. In most patients, PAAD is unresectable after diagnosis (1). According to National Institutes of Health statistics, regardless of tumor stage, the 5-year survival rate of patients with PAAD is only $10 \%$ based on cases of PAAD-associated mortality between 2010 and 2016 (2). PAAD exhibits a limited response to traditional therapy approaches; however, a number of novel therapies, including immunotherapy, have been tested in the clinical trial phase (3). Unfortunately, the immunosuppressive tumor microenvironment (TME) limits the efficacy of immunotherapy (4). Therefore, the discovery of novel biomarkers that characterize TMEs that would be suitable candidates for immunotherapy is urgently required for the prognostic assessment of patients with PAAD (5).

UDP-GlcNAc: $\beta$ Gal $\beta-1,3-N$-acetylglucosaminyltransferase 3 (B3GNT3) is a type II transmembrane protein on the Golgi membrane that acts as the catalytic center in the synthesis of poly-N-acetyllactosamine chains and generation of the backbone components of dimeric sialyl Lewis A (6). Additionally, this gene serves vital roles in L-selectin ligand synthesis, which is involved in lymphocyte homing and trafficking. Due to its biological characteristic, B3GNT3 has been considered to be involved in the tumorigenesis of non-Hodgkin's lymphoma $(7,8)$. B3GNT3 expression is tissue-selective. It is aberrantly expressed in the pancreas and distributed throughout the gastrointestinal tract, liver, placenta, kidney, trachea, neutrophils and lymphocytes $(9,10)$. However, to the best of our knowledge, the role of B3GNT3 in the tumorigenesis of PAAD has not been fully revealed.

Currently, the molecular function of B3GNT3 is controversial. Ho et al (11) reported that B3GNT3 is an independent predictor for a good prognosis of neuroblastoma via the suppression of extend core (T-antigen) oligosaccharide formation. However, other researchers have revealed that B3GNT3 has a negative role in cancer. For example, Zhang et al (12) claimed that increased expression levels of B3GNT3 were associated with pelvic lymph node metastasis and poor prognosis in patients with early-stage cervical cancer. Furthermore, Gao et al (13) demonstrated that, among patients who suffer from non-small cell lung cancer, patients with high B3GNT3 expression have worse disease-free survival (DFS) time and overall survival (OS) time. According to another study, B3GNT3 is essential for 
the epidermal growth factor-induced communication of receptor programmed cell death protein-1 (PD-1) and programmed death-ligand 1 in triple-negative breast cancer (14). Therefore, the downregulation of B3GNT3 may enhance cytotoxic T cell-mediated anti-neoplastic effects. Overall, B3GNT3 may have tumor-promoting and tumor-suppressive effects.

In the present study, The Cancer Genome Atlas (TCGA) was used to analyze the immunohistochemical (IHC) results of samples from a single center to evaluate the prognostic value of B3GNT3 expression in PAAD. Gene Set Enrichment Analysis (GSEA) was used to gain further insights into the biological pathways involved in the pathogenesis of PAAD. Furthermore, the association between B3GNT3 and tumor-infiltrating immune cells in the TME was examined to identify a probable immunotherapy target in PAAD.

\section{Materials and methods}

Data source and clinical information. The gene expression profiles of PAAD (171 cases; workflow type, HTSeq-Counts), and the relevant clinical information and pathologic characteristics were downloaded from TCGA (https://portal.gdc.cancer. gov; Project ID, TCGA-PAAD; Table I).

IHC analysis. To assess the protein expression patterns of B3GNT3 and CD68, IHC staining was performed on 177 paraffin-embedded PAAD samples (collected from the Department of Pathology, Changhai Hospital, Navy Medical University, Shanghai, China). The inclusion criteria were as follows: i) Histological type of adenocarcinoma; and ii) treatment by surgery. Among 177 cases, 115 were male and 62 were female. The age range was $32-86$ years with a mean age of $60.98 \pm 10.75$ years and a median age of 62 years. All data were collected between July 2018 and December 2019. The IHC procedures abided by established protocols (12); however, 16 tissue dots on the microarrays were missed during the procedure. In brief, fresh tissues were fixed in $10 \%$ formalin for $48 \mathrm{~h}$ at room temperature and then dehydrated in ethanol, cleared in xylene for transparence and embedded in molten paraffin. The paraffin-embedded tissue block was sectioned into 4- $\mu \mathrm{m}$ thick slices using a microtome (Leica Microsystems, Inc.). Paraffin sections were heated, dewaxed in xylene and hydrated in different concentrations of ethanol $(100,95,85$ and $70 \%)$ and washed in PBS buffer (15). The sections were submerged in a high-pressure cooker filled with EDTA antigenic retrieval buffer and heated at $110^{\circ} \mathrm{C}$ for $5-10 \mathrm{~min}$. Endogenous peroxidase activity was inhibited by incubation with $3 \%$ hydrogen peroxide for $25 \mathrm{~min}$, followed by incubation with goat serum working fluid (undiluted; cat. no. ZLI-9056; OriGene Technologies, Inc.) to block the non-specific protein binding for $30 \mathrm{~min}$ at $25^{\circ} \mathrm{C}$. Subsequently, the specimens were coated with polyclonal antibodies against B3GNT3 (dilution, 1:100; cat. no. 18098-1-AP; ProteinTech Group, Inc.) and CD68 (dilution, 1:800; cat. no. 76437; Cell Signaling Technology, Inc.) (16), and incubated overnight at $4^{\circ} \mathrm{C}$. PBS replaced the primary antibodies as a negative control. After three washes with PBS with $0.2 \%$ Tween-20, the tissue slices were incubated with a biotinylated anti-rabbit/mouse secondary antibody working fluid (undiluted; cat. no. PV8000-1; OriGene Technologies, Inc.) at room temperature $\left(25^{\circ} \mathrm{C}\right)$ for $30 \mathrm{~min}$. For visual staining, 3,3-Diaminobenzidine (cat. no. ZLI-9017; OriGene Technologies, Inc.) was dripped on the sections. The tissue
Table I. Clinical information from TCGA database (project ID, TCGA-PAAD).

\begin{tabular}{|c|c|c|}
\hline Clinical characteristics & Total, $\mathrm{n}(\mathrm{n}=171)$ & Percentage \\
\hline \multicolumn{3}{|l|}{ Sex } \\
\hline Male & 93 & 54.4 \\
\hline Female & 78 & 45.6 \\
\hline \multicolumn{3}{|l|}{ Age, years } \\
\hline$\leq 60$ & 57 & 33.3 \\
\hline$>60$ & 114 & 66.7 \\
\hline \multicolumn{3}{|l|}{ T stage } \\
\hline $\mathrm{T} 1$ & 7 & 4.1 \\
\hline $\mathrm{T} 2$ & 21 & 12.3 \\
\hline $\mathrm{T} 3,4$ & 141 & 82.5 \\
\hline \multicolumn{3}{|l|}{$\mathrm{N}$ stage } \\
\hline N1 & 47 & 27.5 \\
\hline No & 119 & 69.6 \\
\hline \multicolumn{3}{|l|}{ M stage } \\
\hline M1 & 77 & 45 \\
\hline M0 & 4 & 2.3 \\
\hline \multicolumn{3}{|l|}{ Grade } \\
\hline G1 & 28 & 16.4 \\
\hline $\mathrm{G} 2 / 3 / 4$ & 141 & 82.5 \\
\hline \multicolumn{3}{|l|}{ Histology } \\
\hline PDAC & 140 & 81.9 \\
\hline Others & 30 & 17.5 \\
\hline \multicolumn{3}{|l|}{$\mathrm{MSI}^{\mathrm{a}}$} \\
\hline MSI-L & 9 & 5.3 \\
\hline MSS & 137 & 80.1 \\
\hline
\end{tabular}

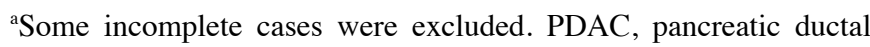
adenocarcinoma; MSI, microsatellite instability; MSS, microsatellite stable; MSI-L, low-frequency MSI.

sections were then washed with running water, counterstained with $10 \%$ Mayer's hematoxylin at room temperature for 1-3 min, dehydrated in anhydrous ethanol and sealed with a coverslip.

The IHC staining results were observed using a confocal microscope (Olympus Corporation) and scored independently by two pathologists blinded to the clinical characteristics in a semi-quantitative manner. The tissue specimens were scored based on the proportion of positive cancer cells and staining strength. A positive reaction was defined as a cell exhibiting brown staining in the cytoplasm. For B3GNT3 expression scoring, the staining index was determined as the staining intensity score $(0$, negative; 1 , weak; 2 , moderate; 3 , strong) multiplied by the score for the positive area $(0,<5 \% ; 1,5-25 \%$; $2,25-50 \% ; 3,50-75 \% ; 4,>75 \%)$. Therefore, the final sores were $0,1,2,3,4,6,8,9$ and 12 . For statistical analysis, the scores of 0 -1 were considered negative; $2-4$ were considered weak; 6 and 8 were considered moderate; and 9 and 12 were considered strong. For immune cells, the proportion of positive cells was counted and the samples were classified into four groups: $0,<5 \% ; 1,5-25 \% ; 2,25-75 \%$; and $3,>75 \%$. 

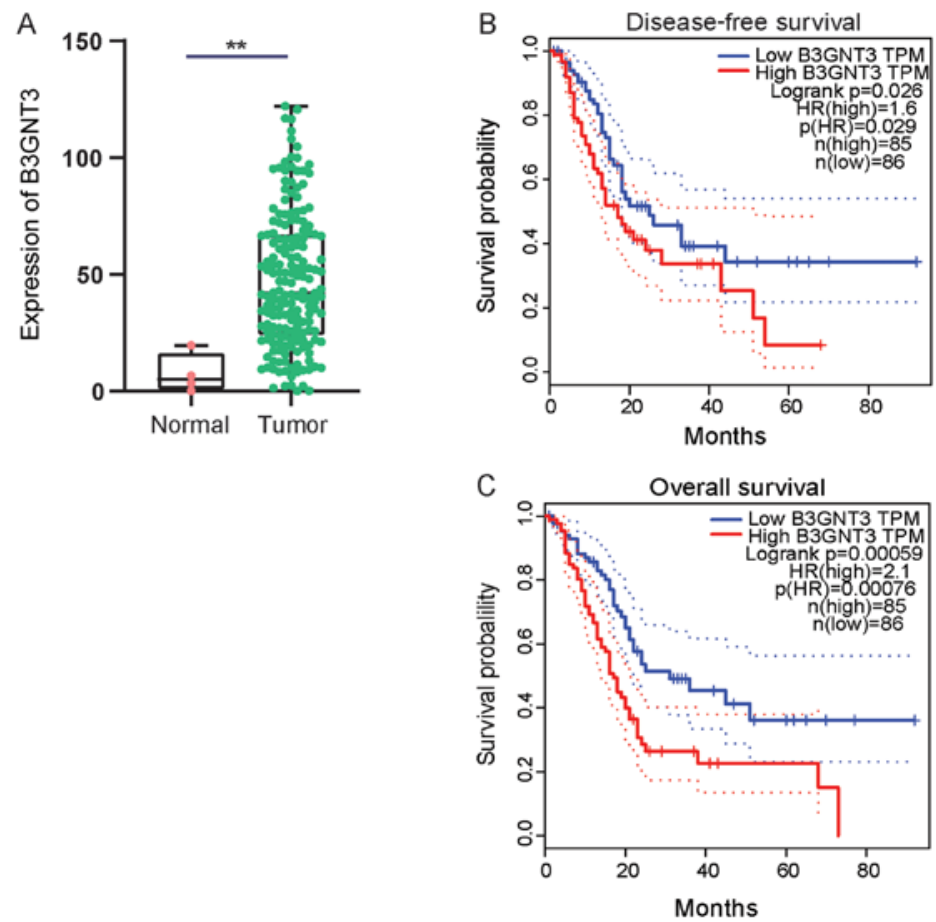

Figure 1. Effect of TFAP2B expression in patients with PAAD in TCGA cohort. (A) B3GNT3 expression in pancreatic cancer (47.45 \pm 29.06$)$ and normal pancreas (7.36 \pm 7.44$)$ samples. Effect of $B 3 G N T 3$ expression on (B) disease-free survival and (C) overall survival in patients with pancreatic adenocarcinoma in the cohort from TCGA ( $\mathrm{n}=171$; overall survival $\mathrm{HR}=2.1, \mathrm{P}=0.00076$; disease-free survival $\mathrm{HR}=1.6, \mathrm{P}=0.029) .{ }^{* *} \mathrm{P}<0.01$. B 3 GNT3, $\beta \mathrm{Gal} \beta-1,3-\mathrm{N}$-acetylgluco saminyltransferase 3; HR, hazard ratio; TPM, transcripts per million.

GSEA. GSEA was performed to investigate the difference in survival between the high and low B3GNT3 expression groups using GSEA software (http://www.broadinstitute. org/gsea; version 4.1.0 for Windows). Gene set permutations were conducted 1,000 times for each analysis. The expression degree of B3GNT3 was used as a phenotype marker. The nominal P-value and normalized enrichment score were used to rank the enrichment pathways in each phenotype.

Tumor Immune Estimation Resource (TIMER) database analysis. The tumor immune cell infiltration characterization of PAAD was estimated using data provided by TIMER web portal (http://cistrome.dfci.harvard.edu/TIMER/). The correlations among B3GNT3 and different immune cells and associated gene markers were explored. The related module generated scatter plots of the expression of a pair of user-defined genes in pancreatic cancer, as well as the Spearman's correlation analysis and the estimated statistical significance.

Tumor-Immune System Interaction Database (TISIDB) analysis. The TISIDB web portal (http://cis.hku.hk/TISIDB) comprises 988 identified immune-associated oncogenes and antitumor genes, high-throughput screening techniques, exome and RNA sequencing data, and a variety of resources for immunological data collected from other public databases. It facilitates the analysis of the interaction of certain genes with immunocytes, immunomodulators and cytokines. In the present study, this database was utilized to investigate the associations between B3GNT3 expression and lymphocytes and immunomodulators $(15,17)$.

Statistical analysis. Clinical data and B3GNT3 expression information were collected from TCGA database and analyzed using R studio (https://cran.r-project.org/; version 1.2.1335) and SPSS (version 23.0.0; IBM Corp). All data are presented as the mean \pm SD. The association between clinicopathologic features and B3GNT3 expression was analyzed using an unpaired t-test. A univariate Cox proportional hazards model was used to evaluate risk factors associated with the survival of patients with pancreatic cancer. Subsequently, clinical parameters with $\mathrm{P}<0.05$ were used in a multivariate Cox proportional hazards model to assess prognostic factors. The OS time associated with B3GNT3 expression was examined by Kaplan-Meier analysis to analyze the diversity between the high- and low-expression groups. The cut-off value of B3GNT3 expression was determined by its median value. The log-rank P-value was also computed. Clinical information, such as survival-associated IHC results, was analyzed using Cox regression analysis. The correlation analyses of B3GNT3 and immune infiltration were based on Pearson's correlation analysis (after tests of normal distribution) and Spearman's regression analysis. While gene set permutations were conducted 1,000 times for each analysis, other analyses were repeated only once. All statistical tests were two-sided, and $\mathrm{P}<0.05$ was considered to indicate a statistically significant difference.

\section{Results}

B3GNT3 expression is associated with survival in PAAD. First, B3GNT3 expression in pancreatic cancer was investigated using TCGA data. A cohort analysis revealed that B3GNT3 expression was significantly higher in pancreatic ductal adenocarcinoma tissues than in normal tissues at the mRNA level (Fig. 1A). Kaplan Meier survival curves were generated to analyze the association between B3G NT3 expression and the prognosis of the pancreatic 
A

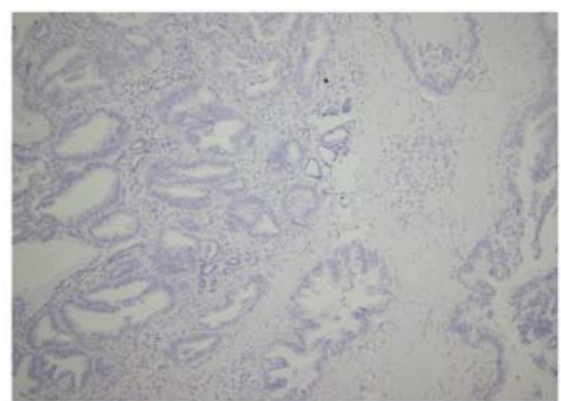

C

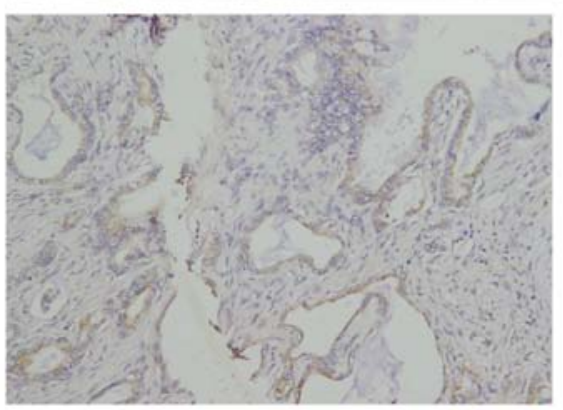

B

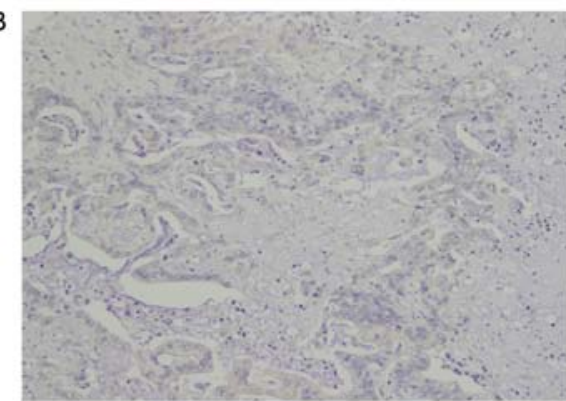

D

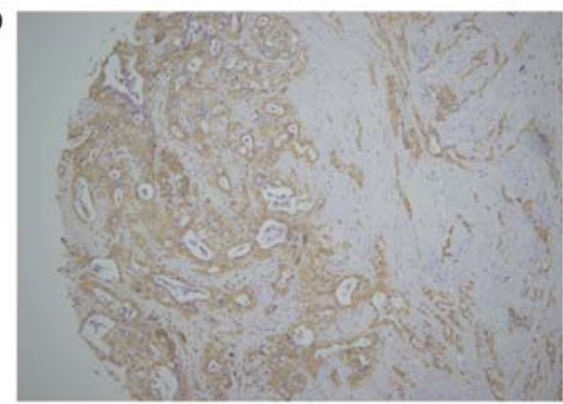

Figure 2. Immunohistochemical analysis of B3GNT3 in pancreatic adenocarcinoma. (A) Negative, (B) weak, (C) moderate and (D) strong B3GNT3 expression in adenocarcinoma tissues. Magnification, x200. B3GNT3, $\beta$ Gal $\beta-1,3-\mathrm{N}$-acetylglucosaminyltransferase 3.

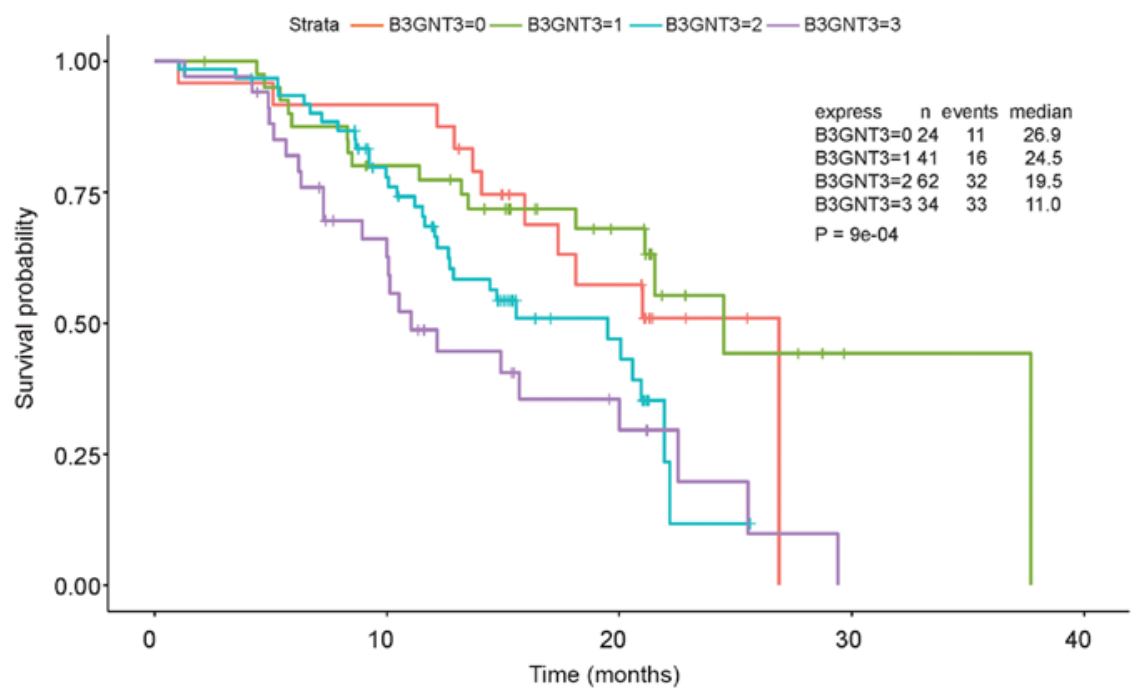

Figure 3. Overall survival of 161 patients with pancreatic adenocarcinoma in relation to B3GNT3 status. Patients with pancreatic cancer with higher B3GNT3 expression had poorer outcomes. The P-value shown in the figure applies to the comparison of all groups. B3GNT3, $\beta$ Gal $\beta-1,3-\mathrm{N}$-acetylglucosaminyltransferase 3 .

cancer cohort using follow-up information. Patients in the high B3GNT3 expression group had a shorter OS time and DFS time [OS: Hazard ratio $(\mathrm{HR})=2.1, \mathrm{P}=0.00076$; DFS: $\mathrm{HR}=1.6, \mathrm{P}=0.029$; Fig. $1 \mathrm{~B}$ and $\mathrm{C}]$.

IHC was performed to estimate B3GNT3 protein expression in a retrospective cohort of 177 pancreatic ductal adenocarcinoma samples, among which 16 cases were censored. Immunoreactivity to the B3GNT3 antibody was detected primarily in the cytoplasm (Fig. 2). The IHC staining of B3GNT3 was positive in 137 cases, among which 41 cases $(30 \%)$ were stained weakly for B3GNT3, 62 (45\%) were stained moderately and 34 (25\%) were stained strongly. Furthermore, Cox regression analysis indicated that the group with the highest expression levels of B3GNT3 had worst outcomes (Fig. 3).
Table II. Associations between overall survival and clinicopathologic characteristics in TCGA patients analyzed using Cox regression analysis.

\begin{tabular}{lccc}
\hline Clinicopathologic variable & OR & P-value & 95\% CI \\
\hline Grade (1 vs. grade 2/3/4) & 2.160 & 0.019 & $1.137-4.104$ \\
Stage (I vs. II/III/IV) & 2.283 & 0.038 & $1.046-4.980$ \\
Topography (T1/2 vs. T3/4) & 2.021 & 0.030 & $1.071-3.815$ \\
MSI (MSI-L vs. MSS/MSI-H) & 0.450 & 0.046 & $0.206-0.985$ \\
B3GNT3 & 2.025 & 0.016 & $1.325-3.093$
\end{tabular}

B3GNT3, $\beta$ Gal $\beta$-1,3-N-acetylglucosaminyltransferase 3; MSI, microsatellite instability; MSS, microsatellite stability; MSI-H, microsatellite instability-high; MSI-L, microsatellite instability-low; OR: Odds ratio. 
Table III. Gene sets enriched in phenotype low.

Gene set

NES NOM P-value FDR q-val

\begin{tabular}{|c|c|c|c|}
\hline GO: CHEMOKINE MEDIATED SIGNALING PATHWAY & -1.89 & 0.006 & 0.16 \\
\hline GO: POSITIVE REGULATION OF NATURAL KILLER CELL-MEDIATED IMMUNITY & -1.76 & 0.005 & 0.14 \\
\hline GO: NEGATIVE REGULATION OF STAT CASCADE & -1.73 & 0.002 & 0.14 \\
\hline
\end{tabular}

Gene sets with NOM P<0.05 and FDR q-val<0.25 were considered to be significant. NES, normalized enrichment score; NOM, nominal; FDR, false discovery rate; GO, Gene Ontology.

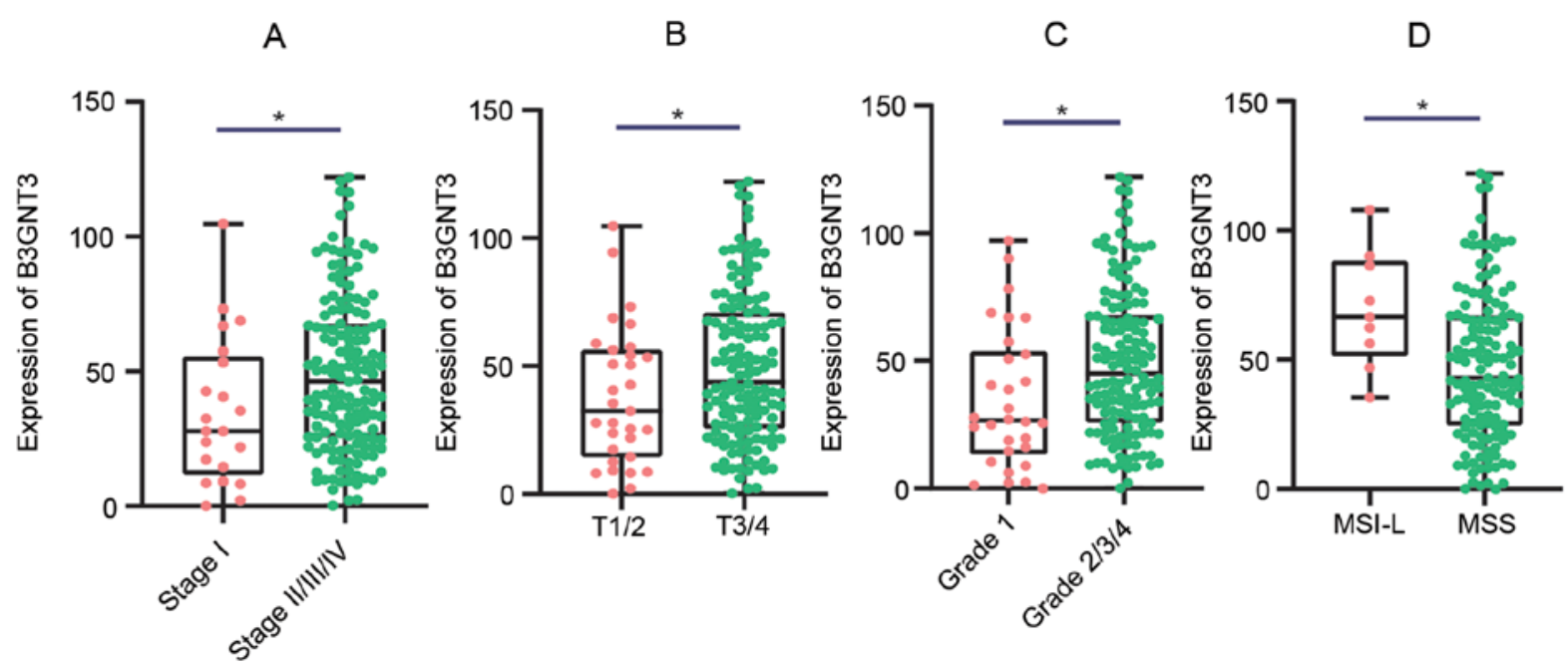

Figure 4. Association between B3GNT3 expression and clinicopathologic characteristics. (A) Clinical stage: Stage 1 (35.09 \pm 26.66$)$ vs. Stage 2/3/4 (49.24 \pm 28.86$)$. (B) Tumor/topography: T1/T2 (37.82 \pm 26.32$)$ vs. T3/T4 (49.64 \pm 29.09$)$. (C) Grade: G1 (34.60 \pm 26.75$)$ vs. G2/3/4 (49.65 28.91$)$. (D) MSI: MSI-L $(69.43 \pm 21.43)$ vs. MSS $(47.69 \pm 28.83)$. B3GNT3 expression was associated with malignant behavior of pancreatic cancer. "P<0.05. B3GNT3, $\beta$ Gal $\beta-1,3-\mathrm{N}-$ acetylglucosaminyltransferase 3; MSI, microsatellite instability; MSS, microsatellite stable; MSI-L, low-frequency MSI.
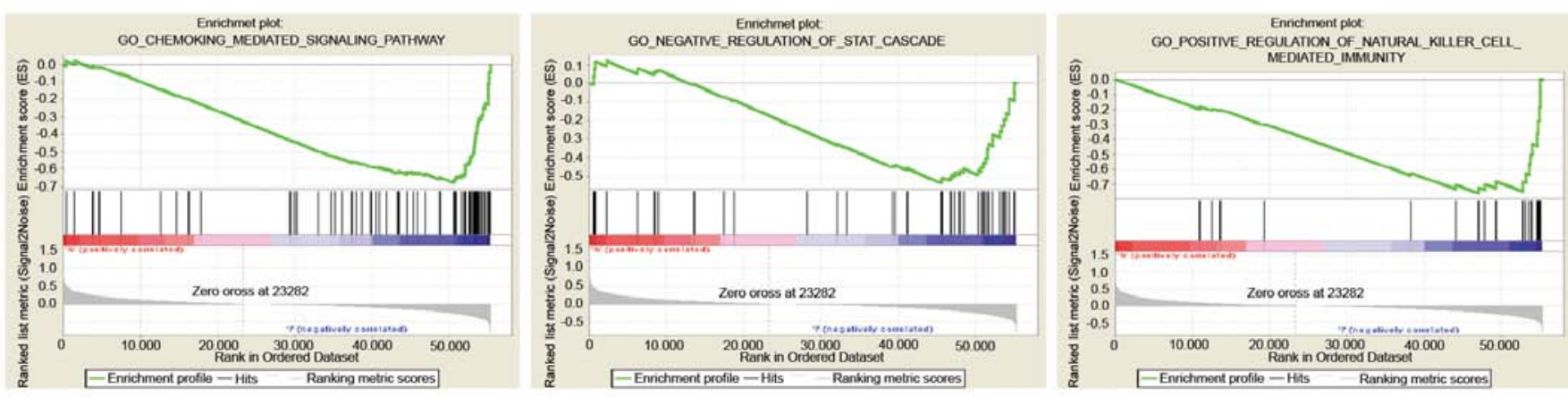

Figure 5. Enrichment plots from Gene Set Enrichment Analysis. The results were analyzed and it was revealed that pathways, such as 'chemokine mediated signaling pathway', 'negative regulation of STAT cascade' and 'positive regulation of natural killer cell mediated immunity', were mostly enriched in pancreatic adenocarcinoma samples with low B3GNT3 expression. B3GNT3, $\beta$ Gal $\beta-1,3-\mathrm{N}$-acetylglucosaminyltransferase 3; ES, enrichment score; GO, Gene Ontology.

B3GNT3 expression is associated with clinicopathologic factors. The association between B3GNT3 expression and the clinicopathological characteristics of patients with PAAD was analyzed using data from TCGA. High B3GNT3 expression in PAAD was significantly associated with intraepithelial neoplasia, tumor/topography (18), stage and microsatellite instability (Fig. 4). The univariate analysis revealed that high expression levels of B3GNT3 were associated with poor OS time in PAAD. Other clinicopathologic variables associated with poor survival included high grade, advanced stage, high levels of tumor/topography and microsatellite instability (Table II).

GSEA identification of a B3GNT3-related signaling pathway. GS EA between the low- and high-expression B3GNT3 datasets was performed to identify differentially activated signaling pathways in pancreatic cancer (Fig. 5; Table III). 


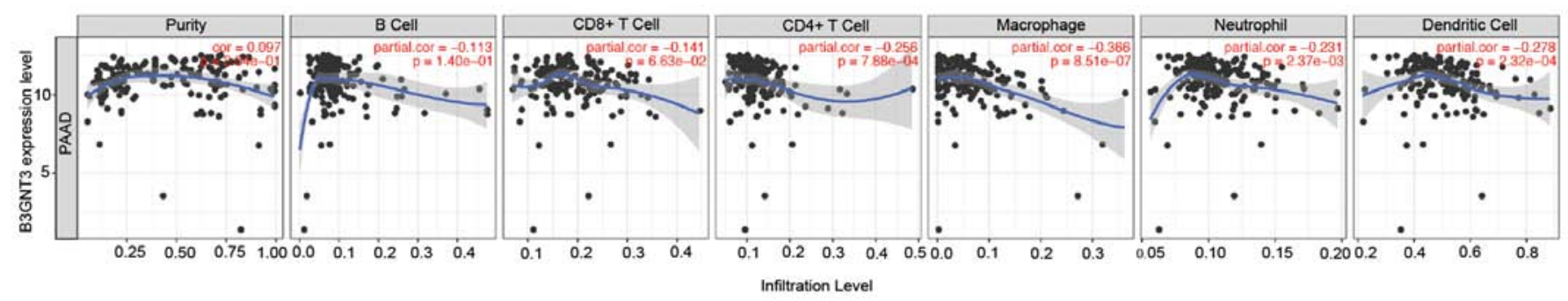

Figure 6. Correlation between $B 3 G N T 3$ expression and tumor immune infiltration levels in PAAD ( $\mathrm{n}=171)$ according to Tumor Immune Estimation Resource database analysis. B3GNT3 expression was negatively correlated with $\mathrm{CD} 4^{+} \mathrm{T}$ cells, neutrophils, macrophages and dendritic cells (|corl $\left.>0.2\right)$. B3GNT3, $\beta \mathrm{Gal}$ $\beta-1,3-\mathrm{N}$-acetylglucosaminyltransferase 3; cor, correlation; PAAD, pancreatic adenocarcinoma.

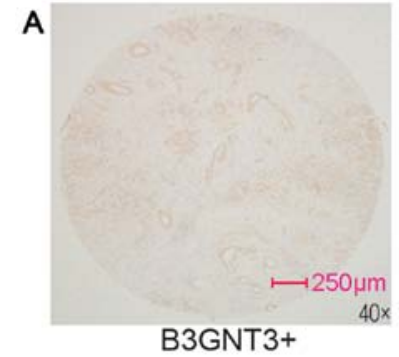

B

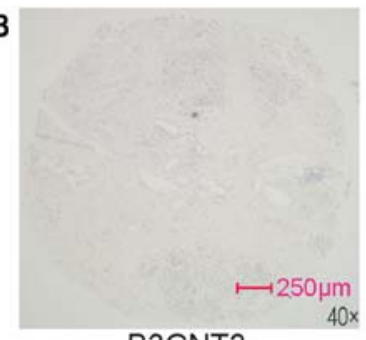

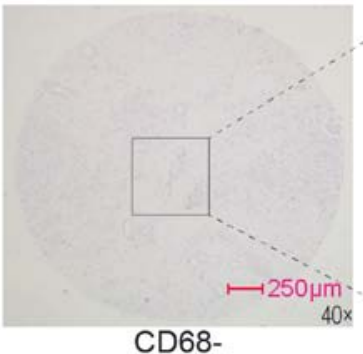

CD68-

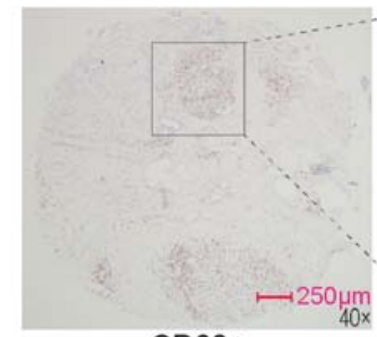

CD68+
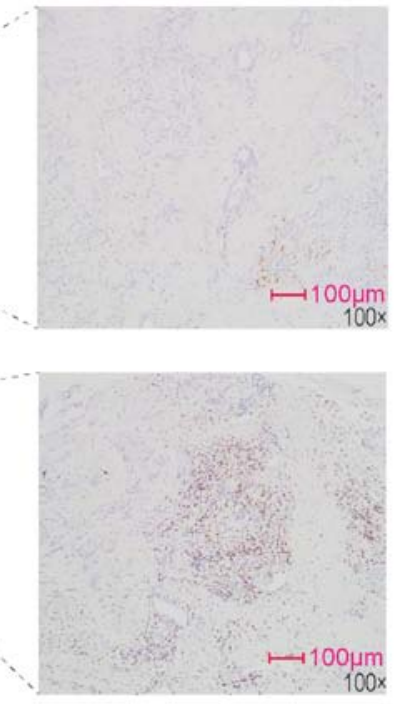

C

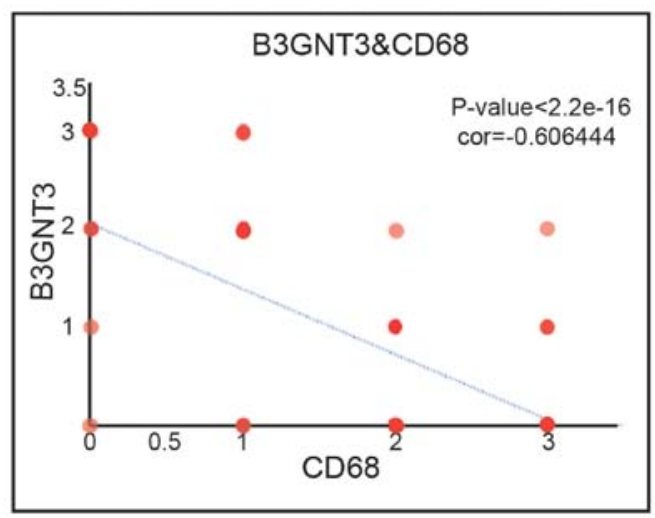

Figure 7. Association of $B 3 G N T 3$ expression with macrophage infiltration levels in PAAD. (A) PAAD tissue exhibiting $B 3 G N T 3^{+}$but $C D 68^{-}$staining. (B) PAAD tissue exhibiting $B 3 G N T 3^{-}$but $\mathrm{CD}^{+} 8^{+}$staining. (C) Spearman regression analysis of $B 3 G N T 3$ expression with macrophage maker $\mathrm{CD} 68$. $\mathrm{P}<0.05$, cor=-0.606444. B3GNT3, UDP-GlcNAc: $\beta$ Gal $\beta-1,3-\mathrm{N}$-acetylglucosaminyltransferase 3; cor, correlation; PAAD, pancreatic adenocarcinoma. 0, negative; 1 , weak; 2 , moderate; 3 , strong.

B3GNT3 expression is associated with immune infiltration levels in PAAD. The association between B3GNT3 expression and immune infiltration levels in pancreatic cancer was investigated by assessing the correlations between B3GNT3 expression and tumor immune infiltration levels using TIMER. B3GNT3 expression and immune infiltration levels, including $\mathrm{CD}^{+} \mathrm{T}$ cells, neutrophils, macrophages and dendritic cells, were negatively correlated in pancreatic cancer (Fig. 6), especially macrophages ( $\mid$ corl=0.366). To support this result, CD68, a maker of macrophages, was assessed by immunohistochemical staining. Spearman's regression analysis was performed to evaluate the correlation of each molecule with B3GNT3 expression. The results revealed that the infiltration of macrophages exhibited a negative correlation with B3GNT3 expression (Fig. 7).

B3GNT3 expression is associated with immune signatures. The correlation between B3GNT3 expression and various immune signatures was examined by TISIDB analysis. The present study focused on tumor-infiltrating immunocytes, 

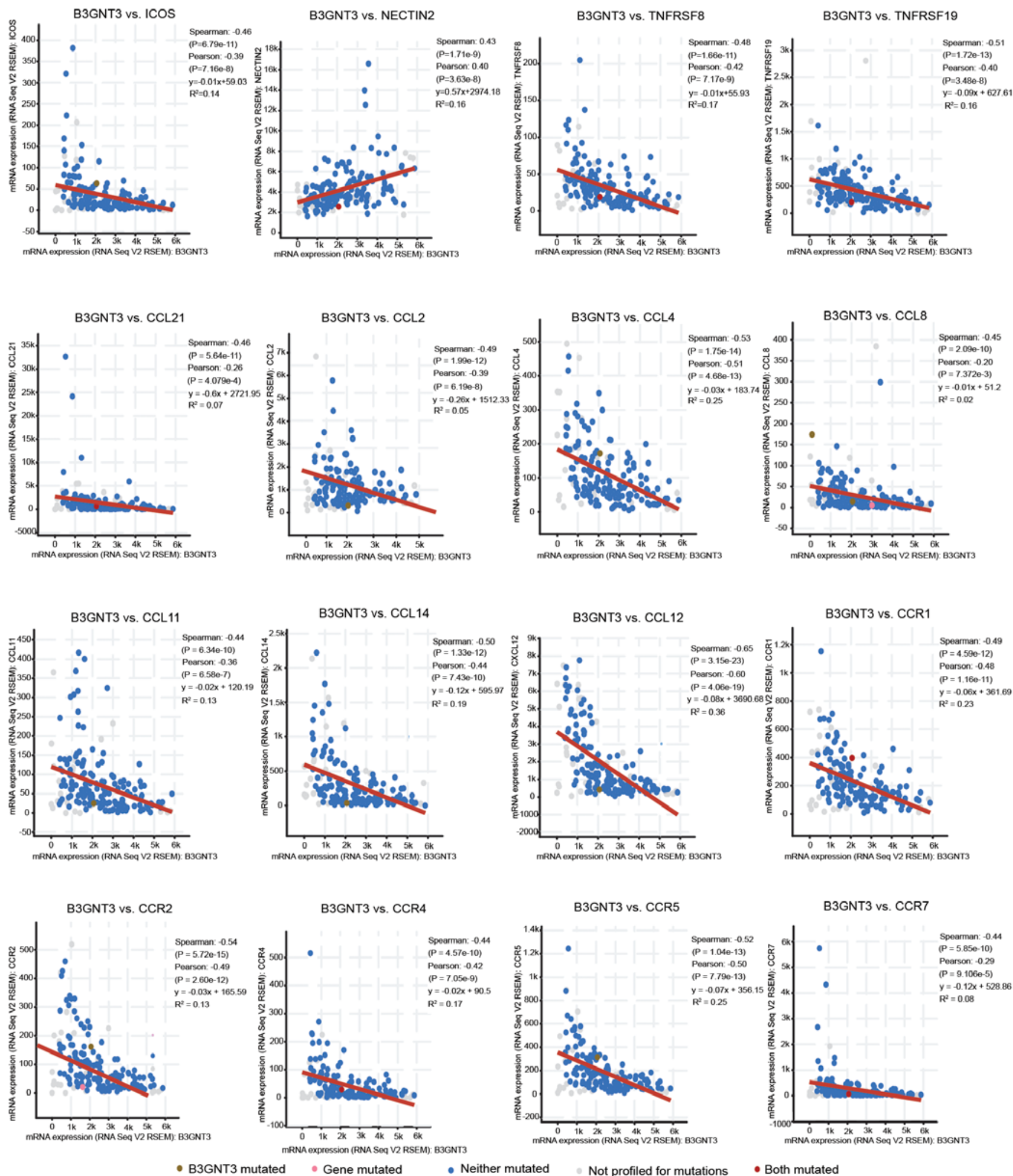

- B3GNT3 mutated - Gene mutated

- Neither mutated

Not profiled for mutations

- Both mutated

Figure 8. Associations between immunomodulators and B3GNT3 expression, and correlations between chemokines (or receptors) and B3GNT3 expression. B3GNT3, $\beta$ Gal $\beta$-1,3-N-acetylglucosaminyltransferase 3; ICOS, inducible co-stimulator; NECTIN2, poliovirus receptor-related 2; TNFRSF, tumor necrosis factor receptor superfamily; CCL, chemokine (C-C motif) ligand; CXCL12, C-X-C motif chemokine ligand 12; CCR, C-C motif chemokine receptor.

immune inhibitory or stimulatory genes (including immune checkpoint gene sets) and cytokine-related genes. In Spearman correlation analysis with filtering for $\mathrm{P}<0.05$ and $I \pm$ rhol $>0.4$, B3GNT3 expression was correlated with a set of immune markers in infiltrating immune cells of pancreatic cancer, such as inducible co-stimulator (ICOS) and NECTIN2 (CD112).

The expression levels of immune checkpoint proteins, such as ICOS, were correlated with B3GNT3 expression. Furthermore, a positive correlation was observed between 
$B 3 G N T 3$ expression and poliovirus receptor-related 2 (NECTIN2) expression. Nevertheless, members of the tumor necrosis factor receptor superfamily (TNFRSF), TNFRSF8 (CD30) and TNFRSF19, exhibited negative correlations with B3GNT3 expression in pancreatic cancer. Specifically, the expression levels of chemokine (C-C motif) ligand (CCL)-2, CCL4, CCL8, CCL14, CCL11, CCL21 and CXC motif chemokine ligand 12 , and associated chemokine receptors, including C-C motif chemokine receptor (CCR)1, CCR2, CCR4, CCR5 and CCR7, were significantly correlated with B3GNT3 expression $(\mathrm{P}<0.001$; Fig. 8). Overall, these results demonstrated the correlation between B3GNT3 and the immune infiltrating cells in PAAD, which suggests that B3GNT3 has an important immune escape role in the TME and can be used as a target for immunotherapy.

\section{Discussion}

Studies have demonstrated that B3GNT3 is involved in non-Hodgkin lymphoma tumorigenesis (7) and the determination of malignant behaviors $(12,13)$. In the present study, high expression levels of B3GNT3 in PAAD were positively associated with poor prognosis and advanced clinicopathological features (e.g., high grade and clinical staging) according to biomolecular informatics analysis of high-throughput RNA profiling sequencing data in TCGA. Univariate analysis revealed that high B3GNT3 expression was associated with poor OS time for PAAD. Furthermore, B3GNT3 expression was negatively associated with OS time in clinical PAAD samples from our center.

To further investigate the role of B3GNT3 in pancreatic cancer, IHC analysis was performed to investigate immune cell infiltration. B3GNT3 expression was associated with macrophage infiltration. Similarly, Cerhan et al (7) reported that B3GNT3 is associated with tumor immunity and inflammation and serves an important role in lymphocyte migration and transport, leading to the survival and metastasis of non-Hodgkin's lymphoma tumor cells. Furthermore, the correlation between B3GNT3 expression and the immune-related genes suggests the potential regulatory function of B3GNT3 in tumor immunity in PAAD. Additionally, the present results revealed that B3GNT3 may inhibit regulatory $\mathrm{T}$ cell (Treg) migration, since the expression levels of B3GNT3 were negatively correlated with the infiltrating levels of Tregs (ICOS and TNF receptor superfamily member). ICOS is a standard T cell co-stimulating molecule that promotes $\mathrm{T}$ cell activation via the activation of PI3K signaling (19). High expression levels of ICOS predict a favorable survival outcome in esophageal squamous cell carcinoma (20), gallbladder cancer (21) and hepatocellular carcinoma (22). In addition, a positive correlation was observed between $B 3 G N T 3$ expression and poliovirus receptor-related 2 (NECTIN2) expression, the inhibitory effect of which is mediated by PVR related immunoglobulin domain containing (23). It has been reported that nectin-2 expression is associated with disease progression and poor prognosis in patients with pancreatic ductal adenocarcinoma (24). Nectin-2 expression is upregulated in breast and ovarian carcinoma and could be a promising target for antibody therapy (25). It has been reported that nectin-2 expression is associated with disease progression and poor prognosis in patients with pancreatic ductal adenocarcinoma (24). GSEA analysis revealed that B3GNT3 might contribute to the chemokine-mediated activity of the PI3K signaling pathway, and indicated the negative correlation between $B 3 G N T 3$ expression and immunostimulator expression. CCL2 is required for tumor-associated macrophages to induce immune evasion (26), to promote cancer cell progression (27) and invasion (28). Furthermore, CCL4 is associated with a $\mathrm{T}$ cell-inflamed phenotype in primary and metastatic pancreatic cancer (29). Therefore, B3GNT3, as a potential unfavorable prognostic maker, might contribute to the low infiltration of immune cells and immunostimulators. For the GSEA analysis, B3GNT3 might downregulate the frequency, rate or extent of natural killer cell- and chemokine-mediated immunity. Additionally, STAT is implicated in a wide range of human cancer types, including pancreatic cancer (30-32). Therefore, B3GNT3 could increase the malignant behavior of PAAD via the regulation of the STAT cascade signaling pathway (33).

In a follow-up study, the investigation of effective immune inhibitors, such as myeloid-derived suppressor cells, tumor-associated macrophages and Tregs, particularly in the early stage of cancer, is required to develop novel immunotherapeutic agents. The present study demonstrated that B3GNT3 might be a potential target for prognostic prediction and immune therapy in patients with pancreatic carcinoma.

\section{Acknowledgements}

Not applicable.

\section{Funding}

The present study was supported by the National Natural Science Foundation of China (grant no. 81972282).

\section{Availability of data and materials}

The datasets used and/or analyzed during the current study are available from the corresponding author on reasonable request.

\section{Authors' contributions}

$\mathrm{JZ}$ and MX conceived and designed the experiments, and $\mathrm{KK}$, LX and YZ carried out the experiments. HJ and LX analyzed the data. KK and $\mathrm{YZ}$ wrote the manuscript. All the authors discussed and suggested the experiments. All authors read and approved the final manuscript.

\section{Ethics approval and consent to participate}

All the human tissues used in the present study were microarrays of clinical samples from the study of national Natural Science Foundation of China ('The Malignant Biological Behavior and Mechanism of Pancreatic Ducted Adenocarcinoma Mediated via a Novel Spliceosome MDA5'; approval no. 81972282). The present study has passed the ethics review by the Committee on Ethics of Medicine, Navy Medical University.

\section{Patient consent for publication}

Not applicable. 


\section{Competing interests}

The authors declare that they have no competing interests.

\section{References}

1. Mizrahi J, Surana R, Valle J and Shroff R: Pancreatic cancer. Lancet 395: 2008-2020, 2020.

2. National Cancer Institute, Bethesda M: SEER cancer stat facts: Pancreatic cancer. Journal. https://seer.cancer.gov/statfacts/html/ pancreas.html. Accessed December 17, 2020.

3. Ngo P, Shanshal M and Rojan A: Immunotherapy in pancreatic cancer and the importance of tumour testing. BMJ Case Rep 13: e235774, 2020

4. Wang S, Li Y, Xing C, Ding C, Zhang H, Chen L, You L, Dai M and Zhao Y: Tumor microenvironment in chemoresistance, metastasis and immunotherapy of pancreatic cancer. Am J Cancer Res 10: 1937-1953, 2020.

5. Christenson E, Jaffee E and Azad N: Current and emerging therapies for patients with advanced pancreatic ductal adenocarcinoma: A bright future. Lancet Oncol 21: e135-e145, 2020.

6. Hennet T, Dinter A, Kuhnert P, Mattu TS, Rudd PM and Berger EG: Genomic cloning and expression of three murine UDP-galactose: Beta-N-acetylglucosamine beta1,3-galactosyltransferase genes. J Biol Chem 273: 58-65, 1998.

7. Cerhan JR, Ansell SM, Fredericksen ZS, Kay NE, Liebow M, Call TG, Dogan A, Cunningham JM, Wang AH, Liu-Mares W, et al: Genetic variation in 1253 immune and inflammation genes and risk of non-Hodgkin lymphoma. Blood 110: 4455-4463, 2007.

8. Yeh JC, Hiraoka N, Petryniak B, Nakayama J, Ellies LG, Rabuka D, Hindsgaul O, Marth JD, Lowe JB and Fukuda M Novel sulfated lymphocyte homing receptors and their control by a Core 1 extension beta 1,3-N-acetylglucosaminyltransferase. Cell 105: 957-969, 2001

9. Shiraishi N, Natsume A, Togayachi A, Endo T, Akashima T, Yamada Y, Imai N, Nakagawa S, Koizumi S, Sekine S, et al: Identification and characterization of three novel beta $1,3-\mathrm{N}$-acetylglucosaminyltransferases structurally related to the beta 1,3-galactosyltransferase family. J Biol Chem 276: 3498-3507, 2001.

10. Haider S, Wang J, Nagano A, Desai A, Arumugam P, Dumartin L, Fitzgibbon J, Hagemann T, Marshall JF, Kocher HM, et al: A multi-gene signature predicts outcome in patients with pancreatic ductal adenocarcinoma. Genome Med 6: 105, 2014.

11. Ho WL, Che MI, Chou CH, Chang HH, Jeng YM, Hsu WM, Lin $\mathrm{KH}$ and Huang MC: B3GNT3 expression suppresses cell migration and invasion and predicts favorable outcomes in neuroblastoma. Cancer Sci 104: 1600-1608, 2013.

12. Zhang W, Hou T, Niu C, Song L and Zhang Y: B3GNT3 expression is a novel marker correlated with pelvic lymph node metastasis and poor clinical outcome in early-stage cervical cancer. PLoS One 10: e0144360, 2015.

13. Gao L, Zhang H, Zhang B, Zhu J, Chen C and Liu W: B3GNT3 overexpression is associated with unfavourable survival in non-small cell lung cancer. J Clin Pathol 71: 642-647, 2018.

14. Li CW, Lim SO, Chung EM, Kim YS, Park AH, Yao J, Cha JH, Xia W, Chan LC, Kim T, et al: Eradication of triple-negative breast cancer cells by targeting glycosylated PD-L1. Cancer Cell 33: 187-201 e110, 2018.

15. Wester K, Wahlund E, Sundstrom C, Ranefall P, Bengtsson E, Russell PJ, Ow KT, Malmström PU and Busch C: Paraffin section storage and immunohistochemistry. Effects of time, temperature, fixation, and retrieval protocol with emphasis on p53 protein and MIB1 antigen. Appl Immunohistochem Mol Morphol 8: 61-70, 2000 .

16. Lucca LE, Lerner BA, Park C, DeBartolo D, Harnett B, Kumar VP, Ponath G, Raddassi K, Huttner A, Hafler DA and Pitt D: Differential expression of the T-cell inhibitor TIGIT in glioblastoma and MS. Neurol Neuroimmunol Neuroinflamm 7: e712, 2020

17. Ru B, Wong CN, Tong Y, Zhong JY, Zhong SSW, Wu WC, Chu KC, Wong CY, Lau CY, Chen I, et al: TISIDB: An integrated repository portal for tumor-immune system interactions. Bioinformatics 35: 4200-4202, 2019.
18. van Roessel S, Kasumova G, Verheij J, Najarian RM, Maggino L, de Pastena M, Malleo G, Marchegiani G, Salvia R, Ng SC, et al: International validation of the eighth edition of the american joint committee on cancer (AJCC) TNM staging system in patients with resected pancreatic cancer. JAMA Surg 153: e183617, 2018.

19. Chen H, Fu T, Suh WK, Tsavachidou D, Wen S, Gao J, Tang DN, He Q, Sun J and Sharma P: CD4 T cells require ICOS-mediated PI3K signaling to increase T-Bet expression in the setting of anti-CTLA-4 therapy. Cancer Immunol Res 2: 167-176, 2014.

20. Hong MH, Shin SJ, Shin SK, Kim DJ, Zo JI, Shim YM, Lee SE, Cho BC, Park SY, Choi YL and Kim HR: High CD3 and ICOS and low TIM-3 expression predict favourable survival in resected oesophageal squamous cell carcinoma. Sci Rep 9: 20197, 2019.

21. Wang J, He M, Shi W, Sha H, Feng J, Wang S and Wang Y: Inducible costimulator (ICOS) enhances the cytolytic activity of cytokine-induced killer cells against gallbladder cancer in vitro and in vivo. Cancer Invest 27: 244-250, 2009.

22. Yang J, Liu J, Chen Y, Tang W, Bo K, Sun Y and Chen J: Investigation of ICOS, CD28 and CD80 polymorphisms with the risk of hepatocellular carcinoma: A case-control study in eastern Chinese population. Biosci Rep 39: BSR20181824, 2019.

23. Whelan S, Ophir E, Kotturi MF, Levy O, Ganguly S, Leung L, Vaknin I, Kumar S, Dassa L, Hansen K, et al: PVRIG and PVRL2 are induced in cancer and inhibit $\mathrm{CD}^{+} \mathrm{T}$-cell function. Cancer Immunol Res 7: 257-268, 2019.

24. Liang S, Yang Z, Li D, Miao X, Yang L, Zou Q and Yuan Y: The clinical and pathological significance of nectin-2 and DDX3 expression in pancreatic ductal adenocarcinomas. Dis Markers 2015: 379568, 2015

25. Oshima T, Sato S, Kato J, Ito Y, Watanabe T, Tsuji I, Hori A, Kurokawa $\mathrm{T}$ and Kokubo T: Nectin-2 is a potential target for antibody therapy of breast and ovarian cancers. Molecular Cancer 12: 60, 2013

26. Yang H, Zhang Q, Xu M, Wang L, Chen X, Feng Y, Li Y, Zhang X, Cui W and Jia X: CCL2-CCR2 axis recruits tumor associated macrophages to induce immune evasion through PD-1 signaling in esophageal carcinogenesis. Mol Cancer 19: 41, 2020.

27. Liu Q, Song J, Pan Y, Shi D, Yang C, Wang S and Xiong B: Wnt5a/CaMKII/ERK/CCL2 axis is required for tumor-associated macrophages to promote colorectal cancer progression. Int J Biol Sci 16: 1023-1034, 2020.

28. He M, Yu W, Chang C, Miyamoto H, Liu X, Jiang K and Yeh S: Estrogen receptor $\alpha$ promotes lung cancer cell invasion via increase of and cross-talk with infiltrated macrophages through the CCL2/CCR2/MMP9 and CXCL12/CXCR4 signaling pathways. Mol Oncol 14: 1779-1799, 2020.

29. Romero JM, Grunwald B, Jang GH, Bavi PP, Jhaveri A, Masoomian M, Fischer SE, Zhang A, Denroche RE, Lungu IM, et al: A four-chemokine signature is associated with a T-cell-inflamed phenotype in primary and metastatic pancreatic cancer. Clin Cancer Res 26: 1997-2010, 2020.

30. Ko HJ and Kim YJ: Signal transducer and activator of transcription proteins: Regulators of myeloid-derived suppressor cell-mediated immunosuppression in cancer. Arch Pharm Res 39: 1597-1608, 2016.

31. Kim BH, Yi EH and Ye SK: Signal transducer and activator of transcription 3 as a therapeutic target for cancer and the tumor microenvironment. Arch Pharm Res 39: 1085-1099, 2016.

32. Wei D, Le X, Zheng L, Wang L, Frey JA, Gao AC, Peng Z, Huang S, Xiong HQ, Abbruzzese JL and Xie K: Stat3 activation regulates the expression of vascular endothelial growth factor and human pancreatic cancer angiogenesis and metastasis. Oncogene 22: 319-329, 2003

33. Scholz A, Heinze S, Detjen KM, Peters M, Welzel M, Hauff P, Schirner M, Wiedenmann B and Rosewicz S: Activated signal transducer and activator of transcription 3 (STAT3) supports the malignant phenotype of human pancreatic cancer. Gastroenterology 125: 891-905, 2003.

This work is licensed under a Creative Commons Attribution-NonCommercial-NoDerivatives 4.0 International (CC BY-NC-ND 4.0) License. 